\title{
Esophageal atresia - gastrointestinal and nutritional complications
}

\author{
Ioana-Valentina Nenciu1, Cristina-Adriana Becheanu2,5, Iulia Florentina Tincu ${ }^{3,5}$, \\ Mihaela Balgradean ${ }^{4,5}$ \\ ${ }^{1}$ Medicover Hospital, Bucharest, Romania \\ 2"Grigore Alexandrescu" Emergency Clinical Hospital for Children, Bucharest, Romania \\ 3"Dr. Victor Gomoiu" Clinical Hospital for Children, Bucharest, Romania \\ 4"MS Curie" Emergency Clinical Hospital for Children, Bucharest, Romania \\ 5"Carol Davila" University of Medicine and Pharmacy, Bucharest, Romania
}

\begin{abstract}
Esophageal atresia (EA) is one of the most common digestive malformations; $50 \%$ of the patients with EA have other congenital anomalies associated. Due to the progress regarding the surgical techniques and the treatments given in the neonatal intensive care units, along with perfecting the nutrition techniques, the concern shifted from mortality to the morbidity and the quality of life of the patients with esophageal atresia. In order to apply specific therapeutic programs, an increased attention is given to the early identification of the complications, which are described from the neonatal period up to the adulthood. The most common are the gastrointestinal, nutritional and respiratory complications. One of the complications with major impact on the evolution of patients with esophageal atresia is gastro-esophageal reflux (GER), found in $50 \%$ of patients with esophageal atresia; GER may be met in adults, even without symptoms. The persistence of GER is associated with increasing incidence of complications such as dysphagia, esophagitis, esophageal strictures, esophageal metaplasia, esophageal carcinoma.
\end{abstract}

Keywords: esophageal atresia, gastro-esophageal reflux, dysphagia

\section{INTRODUCTION}

Esophageal atresia, defined as discontinuity between the proximal and the distal esophagus, is one of the most common digestive malformations, with a global incidence of 1 in 2,400 to 4,500 births (1). EA is 2-3 times more frequent in twins (2). Tracheoesophageal fistula (TEF) - congenital communication between the trachea and esopghagus, is usually met in patients with EA. In relation with this association, EA is classified in EA with distal TEF (85\%), EA with proximal TEF (3\%), EA with both proximal and distal TEF $(<1 \%)$, EA without TEF $(8 \%)$ and TEF without EA $(3 \%)$. EA is commonly associated with other malformations, association of congenital anomalies, or syndromes $(2,4,5)$.

The first successful surgical intervention on EA was made by Cameron Haight in $1941(1,6)$. Due to the progress regarding the surgical techniques and the treatments given in the neonatal intensive care units, along with perfecting the nutrition techniques, the concern shifted from mortality to the morbidity and the quality of life of the patients with esophageal atresia (1).

While in the 1950s and 1960s the neonatal mortality rate was $33 \%$, with bronchopneumonia being the first cause of death in EA patients, nowadays the mortality is below $10 \%(4,7)$. The survival of the newborns with birth weight $<1,500 \mathrm{~g}$ and severe cardiac anomalies is $80 \%$, and the patients with low risk have a survival rate up to $98 \%$. Clinical studies suggest that twins have a higher incidence of multiple congenital anomalies and a higher mortality, compared with singletons (9).

EA is a complex pathology, given the associated anomalies and the complications that are met in the evolution. 


\section{ESOPHAGEAL ATRESIA GASTROINTESTIONAL AND NUTRITIONAL ASSOCIATED ANOMALIES}

The complications frequently met in the patients with EA are nutritional, gastrointestinal and respiratory. The predictors for complications in the first year of life are orotracheal intubation before surgery, birth weight $<2,500 \mathrm{~g}$, anastomotic leak and the lack of oral nutrition in the first month (10), CPR at birth, long-gap atresia, prematurity, two-step surgery and hospitalization $>30$ days (8).

A study on 93 patients born in Belgium between 1993 and 2013 revealed the prematurity as the most important risk factor for both gastrointestinal and respiratory complications during the first year of life. Between 1 and 6 years of life VACTERL diagnosis was the significant associated factor with gastrointestinal complications, whilst congenital cardiac anomalies were associated with respiratory complications. The same study stated that $72 \%$ of the children with complications during the first year of life developed complications afterwards (8).

Nutritional and gastrointestinal complications are met since the first year of life, and with the increasing survival, are described up to the adulthood. The most common short- and long-term complications are gastro-esophageal reflux (GER), peptic esophagitis, gastric metaplasia, Barret esophagus, anastomotic strictures, feeding disorders, dysphagia, esophageal dysmotility. In the adults esophageal adenocarcinoma and epidermoid carcinoma have been reported (1).

\section{Gastro-esophageal reflux (GER)}

It is described in half of the EA/TEF patients. Several factors are incriminated: 1 . delayed gastric emptying and antral hypomotility- both commonly met in patients with EA, 2. abnormal esophageal motility, leading to abnormal bolus clearance, with dysphagia and mucosal damage, esophagitis and possible esophageal cancer, 3. surgical repair and gastrostomy, leading to alteration of the anatomical gastro-esophageal junction and the angle of Hiss, 4. surgical repair causes deficient arrangement of the muscle layers and innervation damage, with impact on the esophageal peristaltic function, 5. tracheomalacia and/or tracheal stenosis induce GER by increasing abdomino-thoracic pressure gradient, as a consequence of negative inspiratory force due to airway obstruction (4).

GER may be met in adults, even without symptoms. The persistence of GER is associated with increasing incidence of complications such as dysphagia, esophagitis, esophageal strictures, esophageal metaplasia, esophageal carcinoma.
In has been demonstrated that GER is a risk factor for respiratory complications in patients with EA: persistent atelectasis, aspiration pneumonia, asthma, chronic lung disease with bronchiectasis, tracheomalacia worsening (5). GER is associated with airway obstruction and/or acute life-threatening episodes (American Academy of Pediatrics proposed to replace this term with BRUE = brief resolved unexplained events); these events are present in patients with proximal GER reaching the larynx and in children with GER in the inferior esophagus causing respiratory symptoms $(1,11)$.

In infants under one year of age non-acid GER is more frequent (up to $89.2 \%$ ), and after one year the acid GER is more frequent $-29.6 \%$ vs $10.8 \%$ (12).

$\mathrm{pH}$ testing methods represent the gold-standard for GER diagnosis: esophageal $\mathrm{pH}$ probe testing, $\mathrm{pH}$-impedance testing and wireless $\mathrm{pH}$ testing. $\mathrm{pH}$ monitoring is used to diagnose GER, to evaluate the severity of GER and to identify the acid reflux associated symptoms. In order to evaluate the symptoms caused by non-acid reflux, $\mathrm{pH}$ impedance testing is useful (1).

Regarding GER treatment, ESPHGAN recommends it from the neonatal period up to the age of 1 , and afterwards depending of GER persistence. Proton pump inhibitor (PPI) or $\mathrm{H} 2$ receptor antagonists treatment is associated with the decrease of gastrointestinal and respiratory symptomatology, and with weight gain. Long-term safety of antacid treatment in EA patients has not been enough studied, with concerns regarding the effect of the acid suppression on microbiota and possible higher risk for gastrointestinal and respiratory infections (1).

Giving the persistence of GER after the age of 2 even in asymptomatic patients, ESPGHAN recommends long term monitoring of EA children, in order to identify the possible complications of GER. The persistence of GER should be evaluated by esophageal pH-metry after stopping PPI (17); long-term endoscopic follow-up is also recommended.

\section{Anastomotic stricture (AS)}

It is the most frequent post-operative complication in EA, occurring in up to $60 \%$ of patients; the incidence is higher in long-gap EA. The patients with AS may have feeding and swallowing difficulties, regurgitation and vomiting, food impaction, cough, drooling, recurrent respiratory infections, poor weight gain (1). Anastomotic dilation is the first-line therapy for AE (15), aiming to maintain an optimal esophageal diameter for oral feeding. Adjuvant treatments are systemic or intralesional corticosteroids, Mitomicyn $\mathrm{C}$ and stents mounting. 


\section{Dysphagia}

It is one of the main causes of feeding difficulties in EA patients (15), with incidence between $21 \%$ and $84 \%(1,11)$. In $96 \%$ of cases it is associated with GER, and acid reflux is incriminated in $74 \%$ of these cases (12). Clinical manifestations are wide, with swallowing difficulties, nausea, epigastric pain and respiratory symptoms or asphyxia.

When dysphagia is suspected, patients should be evaluated with contrast studies and with endoscopy with biopsies, in particular in cases with dysphagia after fundoplication, in order to identify mechanical complications. Giving the possible association of vascular abnormalities leading to dysphagia, dyspnea and cyanosis by exterior compression, ESPGHAN recommends exclusion of congenital vascular malformations by chest CT or MR angiograpy. Dysphagia treatment is addressed to underlying cause.

\section{Feeding disorders}

These disorders, listed in Table 1, are frequent after EA surgery. They are caused by esophageal, oropharyngeal and behavioral disorders. Medical and psychological causes of feeding disorders in EA patients are also listed in Table 1.

Events such as vomiting, cough, chocking, food impaction and prolonged mealtimes may influence the child's ability and willingness to eat. Children with long-term gavage feedings may lose the instinct to want to eat because they do not make connection between being hungry and eating $(13,14)$.

TABLE 1. Feeding disorders in EA patients and their causes (4)

\begin{tabular}{|l|l|}
\hline \multicolumn{1}{|c|}{ Feeding disorders in EA patients } & $\begin{array}{l}\text { The causes of feeding } \\
\text { disorders in EA patients }\end{array}$ \\
\hline Aberrant feeding behavior & GER \\
Delayed introduction of solid foods & Dysphagia \\
Selective eating & Aspiration \\
Food refusal & Anastomotic stricture \\
Lengthy mealtimes/slow eating & Esophageal dysmotility \\
Regurgitation & Respiratory complications \\
Food impaction & Behavioral issues \\
Coughing/chocking during meals & \\
Vomiting during meals & \\
Texture avoidance & \\
\hline
\end{tabular}

\section{Esophageal dysmotility}

It affects distal esophagus. Contrast studies reveal abnormal coordination of esophageal contractions (2).

\section{Nutritional status}

It is highly impacted in EA patients. Repeated surgery, feeding disorders, gastrointestinal and respira- tory complications, genetic syndromes and congenital malformations associated have a long-term impact on staturo-ponderal development. Children with EA have a significant reduction of height and weight (mean height Z-score $-1.78 \pm 1.7$, mean weight for height $-1.1 \pm 0.9)(15,16)$. A study conducted by EAT (The Federation for Esophageal Atresia and Tracheo-Esophageal Fistula Support groups), including 928 patients from 25 countries, revealed that children under 18 years old had the weight and the height under the median for age. The same study revealed that only $50 \%$ of patients under 5 years say that they can eat any type of food, whilst $75 \%$ of the children after the age of 5 can eat "everything". Only $35 \%$ of patients under 10 with long-gap atresia can eat without restrictions (17).

\section{Esophagitis, Barret esophagus and esophageal cancer}

Whilst the incidence of respiratory manifestations decrease with the transition to adulthood, part of gastrointestinal symptomatology persists: dysphagia affects between 39 and $85 \%$ of adults (18), GER has a higher prevalence than in the general population.

Beside these digestive complications, the adult patient with EA may develop esophagitis and Barret esophagus, with a higher incidence than the general population (19). Esophageal cancer remains a concern regarding EA patients outcome, even if the studies showed no increase incidence of esophageal cancer in those adults (1).

A study conducted in Finland, including 693 patients treated between 1947-2015, revealed no intestinal metaplasia, epithelial dysplasia or cancer at endoscopic follow-up up to 30-37 years distance from primary anastomosis ( $>2$ successive endoscopies) (20).

ESPGHAN recommends the surveillance of adult patients with EA, with special reference to presence of dysphagia, GER, respiratory symptoms or anemia.

\section{CONCLUSIONS}

Esophageal atresia is a complex pathology due to both genetic anomalies and syndromes that can be associated, and nutritional, gastrointestinal and respiratory complications that may be encountered in evolution, from the neonatal period up to the adulthood.

Despite the progresses regarding operatory techniques and post-operative treatments given, the upcoming complications are frequent and complex, with major impact on patient's quality of life and also on the quality of life of the patient's families. These pro- 
gresses lead to an increased survival, with subsequent complications up to adulthood.

The patient care and follow-up by multidisciplinary teams represent important measures in order to

\section{REFERENCES}

1. Krishnan U, Mousa H, Dall'Oglio L, Homaira N, Rosen R, Faure C, Gottrand F. ESPGHAN-NASPGHAN Guidelines for the Evaluation and Treatment of Gastrointestinal and Nutritional Complications in Children With Esophageal Atresia - Tracheoesophageal Fistula. JPGN. 2016;63:550-70.

2. Spitz L. Oesophageal atresia. Orphanet J Rare Dis. 2007;2:24.

3. Marseglia L, Manti S, D'Angelo G, Gitto E et al. Gastroesophageal Reflux and Congenital Gastrointestinal Malformations. World J Gastroentero. 2015;21:8508-15.

4. Traini I, Menzies J, Hughes J et al. Oesophageal atresia: The growth gap. World J Gastroentero. 2020;26(12):1262-1272.

5. Blanco AJ, Gutierrez Velez A, Solis-Garcia G, Salcedo Posadas A et al. Comorbidities and course of lung function in patients with congenital esophageal atresia. Arch Argent Pediatr. 2020;118(1):2530.

6. Okuyama H, Tazuke $\mathrm{Y}$, Uenoa T et al. Long-term morbidity in adolescents an young adults with surgically treated esophageal atresia. Surg Today. 2017;47:872-76.

7. Ijsselstijn H, Gischler SJ, Toussaint L, Spoel M et el. Growth and development after oesophageal atresia surgery: Need for long-term multidisciplinary follow-up. Paediatr Respir Rev. 2016;9:34-38.

8. Rayyan M, Embrechts M, Van Veer $\mathrm{H}$ et al. Neonatal factors predictive for respiratory and gastro-intestinal morbidity after esophageal atresia repair. Pediatr Neonatol. 2019;60:261-269.

9. Tan Tanny S, Fearon E, Hawley A. Predictors of mortality after primary discharge from hospital in patients with esophageal atresia. J Pediatr. 2020;219:70-75.

10. Castilloux J, Noble AJ, Faure C. Risk factors for short- and long-term morbidity in children with esophageal atresia. J Pediatr. 2010;156:75560. avoid underdiagnosis of associated problems, to reduce the bad effects of the complications and to increase the patient's quality of life.

Conflict of interest: none declared Financial support: none declared

11. Legrand C, Michaud L, Salleron J et al. Long-term outcome of children with oesophageal atresia type III. Arch Dis Child. 2012;97:808-11.

12. Catalano P, Di Pace MR, Caruso AM, et al. Gastroesophageal reflux in young children treated for esophageal atresia: evaluation with pH-multichannel intraluminal impedance. J Pediatr Gastroenterol Nutr. 2011;52:686-90.

13. Ramsay M, Birnbaum R. Feeding difficulties in children with esophageal atresia: Treatment by a multidisciplinary team. Dis Esophagus. 2013;26:410-412.

14. Lees MC, Bratu I, Yaskina M et al. Oral feeding outcomes in infants with esophageal atresia and tracheoesophageal fistula. J Pediatr Surg. 2018;53:929-932.

15. Gottrand M, Michaud L, Sfeir Roni, F. Motility, digestive and nutritional problems in esophageal atresia. Paediatr Resp Rev. 2016;19:28-33.

16. Masuya R, Kaji T, Mukai M. Predictive factors affecting the prognosis and the complications of 73 consecutive cases of esophageal atresia at 2 centers. Pediatr Surg Int. 2018;34:1027-1033.

17. Svoboda E, Fruithof J, Widenmann-Grolig A et al. A patient led, international study of long term outcomes of esophageal atresia: EAT 1. J Pediatr Surg. 2018;53:610-615.

18. Sistonen SJ, Koivusalo A, Nieminen U et al. Esophageal morbidity and function in adults with repaired esophageal atresia with tracheoesophageal fistula: A population- based long-term follow-up. Ann Surg. 2010;251:1167-73.

19. Singh A, Middlesworth W, Khlevner J. Surveillance in patients with esophageal atresia/ tracheoesophageal fistula. Curr Gastroenterol Rep. 2017;19:4.

20. Koivusalo AI, Sistonen SJ, Lindahl HG et al. Long-term outcomes of oesophageal atresia without ot with proximal tracheoesophageal fistula - Gross types A and B. J Pediatr Surg. 2017;52:1571-1575. 